\title{
Take your time
}

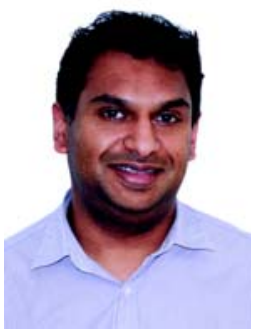

Astika Kappagoda Deputy Edito mja@mja.com.au

doi: 10.5694/mjal3.c1118 ithout the accoutrements of fame, American actress Angelina Jolie would be just one of many young women who are using genetic

knowledge to manage their breast and ovarian cancer risk. However, with her unavoidable celebrity, Jolie's explanation of her preventive double mastectomy, and possible later oophorectomy, to reduce the risk associated with a BRCA1 mutation (http://www.nytimes.com/2013/ 05/14/opinion/my-medical-choice.html) has again brought breast and ovarian cancer to public attention.

In its essentials, Jolie's story is an exercise in the clinical management of genetic information to better one's future health. In such situations, both doctors and patients need time (an often scarce commodity) to negotiate the complex pathway from knowledge to clinical action, as our understanding of diseases and their associations becomes more intricate, but is still incomplete.

Despite the cultural power of a famous actress's real-life story in creating positive effects on health behaviour in society, the complexities of clinical interpretation and practice - and how they affect patients' decisions - can be unintentionally sidelined in public discussion.

Following the surge of media interest in Jolie's announcement, referrals to two familial cancer centres in Victoria almost immediately doubled, according to James and colleagues (page 646). Many of these people had family histories suggesting carriage of a relevant mutation, and among them were probably people at risk who may not otherwise have presented for genetic testing and counselling. Some good may indeed have come from the burst of publicity. But the complex discussion and decision making involved - requiring a concurrent understanding of disease risk, genetics and oncogenesis, and its nuanced application to an individual's circumstances - likely caught many of those presenting to the clinics off guard. Among women with known BRCA1 and BRCA2 mutations, there is currently quite low uptake of preventive options, as research presented by Collins and colleagues (page 680) shows. They propose reasons for this, but it is uncertain what proportion of women not undertaking preventive measures make a fully informed decision not to act, and what proportion are not treated because of an unintended gap in care.

With continuing advances in the field, it is timely to discuss the current application of germline genetics to cancer more generally. Winship and Tucker (page 644) provide an overview of our genetic knowledge about many cancers, and its interpretation and application to clinical decisions, which is now mature enough to be part of routine care. Informed patient counselling requires significant investment of time and effort. Current and future developments, especially in genomics and nextgeneration genetic sequencing, bring ethical and social challenges as well as clinical ones. The old idea that genes would provide clear answers has certainly gone.

Other clinical problems also demonstrate the intersection of incomplete knowledge, problems in diagnostic capability and interpretation, and imperatives to act on the information we have. In a letter to the Editor, MacLachlan and Cowie (page 655) propose that low vitamin D levels increase the likelihood of reactivation of tuberculosis (TB), citing the coincident seasonality of active TB cases and vitamin D deficiency. They advocate vitamin $\mathrm{D}$ testing and supplementation in groups at high risk. In an editorial, Truswell (page 641) outlines plausible physiological reasons for this observation, which may explain the use of sunshine and cod liver oil for treating patients with TB in the sanatoria of old. But, as Ralph and Lucas argue (page 648), many questions remain unanswered about accurate vitamin D testing and interpretation, the benefits of supplementation, and potential harms of oversupplementation. Should we wait for a large-scale randomised controlled trial examining the effects on TB of treating vitamin D deficiency to make a public health recommendation? Or can we act on less definitive evidence and, if so, what level of evidence should that be?

Proper planning for a "good death" for those with increasingly debilitating chronic illness needs to be calm, careful and mindful of the patient's relationships, values and specific wishes, and not devised "on the fly" in a health crisis. Sadly, the reality is that in many cases timely planning does not take place. Scott and colleagues outline the many positive clinical and psychological benefits of advance care planning, and ways to overcome obstacles (page 662). Clinicians should be given proper opportunity to develop advance care plans with patients; even a little more time out of a busy schedule would go a long way. For the community to accept that everyone should allow for such planning as an essential part of their later years, perhaps we now need celebrities to publicly and articulately talk about their own advance care plans. Ultimately, we all need to realise the supreme importance of time, not only for advance care planning, but also for wellbeing - time to discover, time to think, time to talk, time to act. 